\title{
The Turkish Version of Multidimensional Assessment of Fatigue and Fatigue Severity Scale is Reproducible and Correlated With Other Outcome Measures in Patients With Systemic Sclerosis
}

\author{
Kevser GÖK, Gizem CENGİZ, Kemal EROL, Salih ÖZGÖÇMEN \\ Department of Physical Medicine and Rehabilitation, Division of Rheumatology, \\ Medical Faculty of Erciyes University, Kayseri, Turkey
}

\begin{abstract}
Objectives: This study aims to evaluate the reproducibility of Turkish versions of multidimensional assessment of fatigue (MAF) and fatigue severity scales (FSS) and the relationship between health related quality of life, disability, and psychological status in patients with systemic sclerosis (SSc).

Patients and methods: A total of 21 female patients (mean age 47.14 \pm 10.39 years; range 18 to 75 years) who met 2013 American College of Rheumatology/European League Against Rheumatism criteria for SSc were evaluated for severity of organ involvement and symptoms. Turkish version of MAF, FSS, and visual analog scale of fatigue were assessed at baseline and after two to three weeks. Level of dyspnea was noted and disability, functional limitation, and quality of life were assessed by health assessment questionnaire, 6-minute walking distance, and short-form 36, respectively.

Results: Ten patients had diffuse and 11 had limited SSc. MAF subscales and FSS had significant correlations with short-form 36 -vitality subscale and 6 -minute walking distance. Intraclass correlation coefficients for FSS and visual analog scale of fatigue were 0.824 (95\% confidence interval, 0.566 0.929 ) and 0.932 (95\% confidence interval, $0.832-0.972$ ), respectively. The intraclass correlation coefficients for MAF subscales changed between 0.916 and 0.968 , except for MAF-timing (intraclass correlation coefficient, 0.404).

Conclusion: Our results revealed that FSS and MAF subscales had high reproducibility and correlated well with quality of life and disability scales which, to some extent, may suggest convergent validity of MAF subscales and FSS in SSc. The incompatible nature and four-choice answering in two items of MAF-timing may be the underlying reason for trivial relationship with other parameters. The Turkish version of MAF and FSS may be used to assess fatigue in patients with SSc.

Keywords: Fatigue; scale; systemic sclerosis; Turkish version.
\end{abstract}

Systemic sclerosis (SSc, scleroderma) is an autoimmune disease which leads to multi-organ involvement through a combined mechanism of inflammation, fibrosis, and vascular damage. ${ }^{1} \mathrm{SSc}$ effects mostly females and is an important cause of disability and loss of health related quality of life (QoL). ${ }^{2,3}$ Fatigue is increasingly recognized in patients with SSc as a debilitating and bothersome symptom which rated similarly to those patients with other forms of rheumatic disorders and worse than in the general population and cancer patients in remission. ${ }^{4,5}$ However, assessment of fatigue in SSc has been generally conducted using single-item rating which causes a limitation. Multidimensional assessment of fatigue (MAF) scale, as well as Fatigue Severity Scale (FSS), may allow more detailed evaluation of fatigue in SSc.

Multidimensional assessment of fatigue is a revised version of Piper Fatigue Scale which was originally developed and tested in oncology patients. The MAF measures four dimensions of fatigue: severity, distress, degree of interference in activities of daily living, and timing. The global index of MAF is calculated by summing the total scores obtained from severity and 
distress subscales as well as averages of degree in interference in activities of daily living and one of the timing items. The item scores in the subscales are carried out on a 1-10 Likert scale except for timing items which score frequency and degree of fatigue in four grades. Highest scores in MAF indicate greater fatigue. The FSS contains nine statements concerning fatigue and is scored on a 7-point Likert scale. The Turkish versions of both MAF and FSS were shown to be valid and reliable in different patient populations. ${ }^{6-8}$

In this study, we aimed to evaluate the reproducibility of Turkish versions of MAF and FSS and the relationship between health related QoL, disability, and psychological status in patients with SSc.

\section{PATIENTS AND METHODS}

A total of 21 female patients (mean age $47.14 \pm 10.39$ years; range 18 to 75 years) who met the 2013 American College of Rheumatology/ European League Against Rheumatism classification criteria ${ }^{9}$ for $\mathrm{SSc}$ were recruited from our outpatient department between July 2015 and December 2015.

The inclusion criteria were to have no previous diagnosis of comorbidities which may increase fatigue severity or no history of using antipsychiatric drugs or membrane stabilizing agents. We excluded patients with a history of neuroendocrine disorders or receiving high dose corticosteroid therapy or biologics like rituximab.

Patients were scrutinized for symptoms and degree of organ involvement. Cutaneous changes were evaluated by using the modified Rodnan skin score ${ }^{10}$ and patients were clinically classified as having diffuse or limited SSc according to the LeRoy clinical criteria. ${ }^{11}$ Also, all patients were evaluated for physical limitations and QoL by performing health assessment questionnaire and Short-Form 36 (SF-36). Patients' dyspnea scale was graded according to the modified Medical Research Council dyspnea scale which includes five grades. ${ }^{12}$ Six-minute walking distance was noted in meters.

Turkish versions of MAF, FSS, and visual analog scale of fatigue (0-10 numeric scale) within the past week were performed at baseline and two-to-three weeks after the first evaluation. During this period, medications used by patients did not change. All clinical evaluations were performed by the same experienced physician. The Medical Faculty of Erciyes University Ethics Committee approved the study protocol and informed consents were obtained from all patients. The study was conducted in accordance with the principles of the Declaration of Helsinki.

\section{Statistical analysis}

All analyses were performed on a personal computer using IBM SPSS for Windows version 20.0 software (IBM Corporation, Armonk, NY, USA). Relationships between parameters were analyzed by Spearman rank correlation coefficients (ICCs). Test-retest reliability was evaluated by intraclass ICCs. The ICC values were interpreted as follows: $>0.75$ was excellent, $0.40-0.75$ was fair to good, and $<0.40$ was poor.

\section{RESULTS}

Ten patients (47.6\%) had diffuse and 11 (52.4\%) had limited SSc. Medical Research Council dyspnea grades were as follows; nine patients (42.9\%): grade I, eight patients (38.1\%): grade II, three patients (14.3\%): grade III, and one patient (4.8\%): grade IV. Patients' demographics and disease data are given in Table 1. All MAF subscales and FSS had moderate to good correlations with disability and QoL items (Table 2). However, SF-36 vitality which was frequently used as an index for fatigue in various studies did not have significant ICCs with MAF subscales and FSS.

The ICC values were 0.824 (95\% confidence interval, 0.566-0.929) and 0.932 (95\% confidence interval, 0.832-0.972) for FSS and visual analog scale of fatigue, respectively, and changed between 0.916 and 0.968 for MAF subscales which indicated a high level of reproducibility. However, the ICC for MAF-timing was lower than other MAF subscales (0.404).

\section{DISCUSSION}

Our results revealed that FSS and MAF subscales have high reproducibility and significant correlation with QoL and disability scales in SSc. These 


\begin{tabular}{|lccc|}
\hline Table 1. Patients' demographic and clinical data & & & \\
\hline & Median & Minimum & Maximum \\
\hline Age (years) & 48.00 & 29 & 71 \\
Body mass index $\left(\mathrm{kg} / \mathrm{m}^{2}\right.$ ) & 28.72 & 17.58 & 40.25 \\
Symptom duration (years) & 5.00 & 0.67 & 30.00 \\
Modified Rodnan skin score & 15.00 & 2.00 & 30.00 \\
Health assessment questionnaire & 0.25 & 0 & 2.00 \\
Short-form 36-vitality & 55.00 & 30.00 & 95.00 \\
Short-form 36-physical component score & 46.87 & 13.75 & 87.50 \\
Short-form 36-mental component score & 58.33 & 33.88 & 98.75 \\
Fatigue severity scale & 4.14 & 1.22 & 7.00 \\
Multidimensional assessment of fatigue-severity & 7.00 & 3.00 & 10.00 \\
Multidimensional assessment of fatigue-distress & 5.00 & 3.00 & 10.00 \\
Multidimensional assessment of fatigue-interference ADL & 4.77 & 1.36 & 9.00 \\
Multidimensional assessment of fatigue-timing & 5.62 & 2.50 & 10.00 \\
Multidimensional assessment of fatigue-global fatigue index & 26.59 & 1.00 & 46.45 \\
Six-minute walking (meter) & 420.00 & 300.00 & 520.00 \\
Visual analog scale of fatigue & 7.00 & 1.00 & 10.00 \\
\hline ADL: Activities of daily living. & & & \\
\hline
\end{tabular}

results, to some extent, may suggest convergent validity of MAF subscales and FSS in SSc.

The vitality subscale of SF-36, which was frequently used as an index measure to evaluate severity of fatigue in various studies, did not have close relationship with MAF subscales and FSS in our study. Although this has been previously reported by Sandqvist et al., ${ }^{13}$ the small sample size of our study may have hampered more robust ICCs which may be considered as a limitation.

Fatigue is a common and disabling symptom in SSc. ${ }^{4,14,15}$ Fatigue is associated with lower QoL and loss of physical functions, increased pain and depressive symptoms and worsening of daily living activities in patients with SSc. ${ }^{15-17}$ Various questionnaires were used to evaluate fatigue in patients with SSc, including SF-36 vitality subscale and Functional Assessment of Chronic Illness Therapy scale, or MAF., ${ }^{4,15}$ All these studies showed that fatigue is an integral part of the clinical picture in $\mathrm{SSc}$ and monitoring fatigue may also be important to assess the efficacy of any treatment in patients with SSc.

Turkish versions of MAF and FSS were shown to be valid and reliable in different patient populations. The validity and reliability of the Turkish version of the FSS has been investigated in patients with multiple sclerosis by Armutlu et al. ${ }^{6}$ and in patients with fibromyalgia by GencayCan and Can. ${ }^{7}$ In these studies, the Turkish version of the FSS was shown as a valid and reliable measure to assess fatigue in multiple sclerosis and fibromyalgia. The validity and reliability of Turkish version of the MAF for the assessment of fatigue has also been shown in patients with chronic musculoskeletal disorders. ${ }^{8}$

The major limitation of our study was the small number of patients with SSc. We excluded patients under treatment with anti-psychiatric

Table 2. Correlation coefficient matrix between multidimensional assessment of fatigue, fatigue severity scale, visual analog scale of fatigue, health assessment questionnaire, Short-Form 36, and six-minute walking distance

\begin{tabular}{lllcccc}
\hline & VAS-F & HAQ & SF-36-vitality & SF-36-PCS & SF-36-MCS & Six-minute walking \\
\hline Fatigue severity scale & $0.518^{*}$ & $0.532^{*}$ & -0.112 & $-0.521^{*}$ & -0.163 & -0.171 \\
MAF-severity & $0.896^{* *}$ & $0.643^{* * *}$ & -0.189 & $-0.612^{* *}$ & -0.069 & -0.053 \\
MAF-distress & $0.716^{* *}$ & $0.763^{* *}$ & -0.084 & $-0.565^{* *}$ & -0.190 & -0.291 \\
MAF-interference activities of daily living & $0.584^{* *}$ & $0.670^{* *}$ & -0.397 & $-0.726^{* *}$ & $-0.515^{*}$ & -0.396 \\
MAF-timing & 0.116 & 0.136 & -0.426 & $-0.457^{*}$ & -0.296 & -0.356 \\
MAF-global fatigue index & $0.855^{* *}$ & $0.749^{* *}$ & -0.278 & $-0.731^{* *}$ & -0.257 & -0.158 \\
Visual analog scale of fatigue & & $0.599^{* *}$ & -0.226 & $-0.599^{* *}$ & -0.214 & -0.068
\end{tabular}

VAS-F: Visual analog scale of fatigue; HAQ: Health assessment questionnaire; SF-36: Short Form 36; PCS: Physical component score; MCS: Mental component score; MAF: Multidimensional assessment of fatigue; " $\mathrm{p}<0.05$; ${ }^{* *} \mathrm{p}<0.01$. 
drugs or membrane stabilizing agents which limited the sample size.

In conclusion, our study showed that FSS and MAF subscales have high reproducibility and moderate to good correlation with QoL and disability scales which, to some extent, may suggest convergent validity of MAF subscales and FSS in SSc. The incompatible nature and fourchoice answering in two items of MAF-timing may be the underlying reason for lower relationships of this item with other parameters. The Turkish version of MAF and FSS may be used to assess fatigue in patients with SSc.

\section{Declaration of conflicting interests}

The authors declared no conflicts of interest with respect to the authorship and/or publication of this article.

\section{Funding}

The authors received no financial support for the research and/or authorship of this article.

\section{REFERENCES}

1. Denton CP. Systemic sclerosis: from pathogenesis to targeted therapy. Clin Exp Rheumatol 2015;33:3-7.

2. Almeida C, Almeida I, Vasconcelos C. Quality of life in systemic sclerosis. Autoimmun Rev 2015;14:108796.

3. Chifflot H, Fautrel B, Sordet C, Chatelus E, Sibilia $\mathrm{J}$. Incidence and prevalence of systemic sclerosis: a systematic literature review. Semin Arthritis Rheum 2008;37:223-35.

4. Thombs BD, Bassel M, McGuire L, Smith MT, Hudson $\mathrm{M}$, Haythornthwaite JA. A systematic comparison of fatigue levels in systemic sclerosis with general population, cancer and rheumatic disease samples. Rheumatology (Oxford) 2008;47:1559-63.

5. Kesikburun B, Kotseoglu BF, Sahin A, Turgay M, Doğan A, Ayhan Öken Ö. The effects of respiratory muscle weakness and pulmonary involvement on functional status, fatigue and health related quality of life in patients with systemic sclerosis. Arch Rheumatol 2015;30:116-23.

6. Armutlu K, Korkmaz NC, Keser I, Sumbuloglu V, Akbiyik DI, Guney Z, et al. The validity and reliability of the Fatigue Severity Scale in Turkish multiple sclerosis patients. Int $J$ Rehabil Res 2007;30:81-5.

7. Gencay-Can A, Can SS. Validation of the Turkish version of the fatigue severity scale in patients with fibromyalgia. Rheumatol Int 2012;32:27-31.

8. Yildirim Y, Ergin G. A validity and reliability study of the Turkish Multidimensional Assessment of Fatigue (MAF) scale in chronic musculoskeletal physical therapy patients. J Back Musculoskelet Rehabil 2013;26:307-16.

9. van den Hoogen F, Khanna D, Fransen J, Johnson SR, Baron M, Tyndall A, et al. 2013 classification criteria for systemic sclerosis: an American College of Rheumatology/European League against Rheumatism collaborative initiative. Arthritis Rheum 2013;65:2737-47.

10. Clements P, Lachenbruch P, Siebold J, White B, Weiner S, Martin R, et al. Inter and intraobserver variability of total skin thickness score (modified Rodnan TSS) in systemic sclerosis. J Rheumatol 1995;22:1281-5.

11. LeRoy EC, Black C, Fleischmajer R, Jablonska S, Krieg T, Medsger TA Jr, et al. Scleroderma (systemic sclerosis): classification, subsets and pathogenesis. J Rheumatol 1988;15:202-5.

12. Bestall JC, Paul EA, Garrod R, Garnham R, Jones PW, Wedzicha JA. Usefulness of the Medical Research Council (MRC) dyspnoea scale as a measure of disability in patients with chronic obstructive pulmonary disease. Thorax 1999;54:581-6.

13. Sandqvist G, Archenholtz B, Scheja A, Hesselstrand $\mathrm{R}$. The Swedish version of the Multidimensional Assessment of Fatigue (MAF) in systemic sclerosis: reproducibility and correlations to other fatigue instruments. Scand J Rheumatol 2011;40:493-4.

14. Bassel M, Hudson M, Taillefer SS, Schieir O, Baron M, Thombs BD. Frequency and impact of symptoms experienced by patients with systemic sclerosis: results from a Canadian National Survey. Rheumatology (Oxford) 2011;50:762-7.

15. Sandusky SB, McGuire L, Smith MT, Wigley FM, Haythornthwaite JA. Fatigue: an overlooked determinant of physical function in scleroderma. Rheumatology (Oxford) 2009;48:165-9.

16. Ibn Yacoub Y, Amine B, Bensabbah R, Hajjaj-Hassouni $\mathrm{N}$. Assessment of fatigue and its relationships with disease-related parameters in patients with systemic sclerosis. Clin Rheumatol 2012;31:655-60.

17. Suarez-Almazor ME, Kallen MA, Roundtree AK, Mayes M. Disease and symptom burden in systemic sclerosis: a patient perspective. $J$ Rheumatol 2007;34:1718-26.

18. Harel D, Thombs BD, Hudson M, Baron M, Steele $\mathrm{R}$. Measuring fatigue in SSc: a comparison of the Short Form-36 Vitality subscale and Functional Assessment of Chronic Illness Therapy-Fatigue scale. Rheumatology (Oxford) 2012;51:2177-85. 